\title{
Sensitive Voltammetric Determination of Mitoxantrone by Using CS-Dispersed Graphene Modified Glassy Carbon Electrodes
}

\author{
Bin Hong ${ }^{1,2}$, Qiong Cheng ${ }^{2 *}$ \\ ${ }^{1}$ Department of Petrochemical Engineering, Changzhou University, Changzhou, China \\ ${ }^{2}$ Department of Biological and Chemical Engineering, Jiaxing University, Jiaxing, China \\ Email: *qcheng_102@163.com
}

Received May 10, 2012; revised June 13, 2012; accepted June 22, 2012

\begin{abstract}
A novel CS-dispersed graphene modified glassy carbon electrode was fabricated. Study electrochemical characteristics of mitoxantrone in the CS-dispersed graphene modified electrode by cyclic voltammetry and other methods, by selecting and optimizing the various parameters to create a new electrochemical method for the determination of mitoxantrone. The linear range of the oxidation peak current is from $6 \times 10^{-10}$ to $1 \times 10^{-6} \mathrm{~mol} / \mathrm{l}$ in this method, after $2.5 \mathrm{mins}$ open-circuit accumulation, the limit of detection is $2 \times 10^{-10} \mathrm{~mol} / \mathrm{l}$. After 10 parallel determinations, the relative standard deviation was $3.7 \%$ that the concentration of mitoxantrone was $1 \times 10^{-8} \mathrm{~mol} / \mathrm{l}$. The modified electrode has been successfully applied for the assay of mitoxantrone in human urine samples.
\end{abstract}

Keywords: Mitoxantrone; CS-Dispersed Graphene; Modified Electrode; Voltammetry

\section{Introduction}

Graphene, a new class of two-dimensional carbon nanostructure, discovered by Geim [1], because of their outstanding electronic transport properties, high mechanical stiffness, and remarkable thermal and electrical conductivity, scientists are interested in graphene recently, due to its fascinating physical properties [1-5] that is previously mentioned. It also has been predicted to have great promise for many potential applications, for instance, sensors, nanoelectronics, batteries, supercapacitors, hydrogen storage, and nanocomposites [6-9]. Carbon materials have been widely used in electroanalytical chemistry, especially in sensors, the electrochemical biosensors utilizing nanomaterials have recently attracted considerable attention in the area of sensing $[10,11]$, have been used in biosensors in the food quality control and medicine field. Graphene has been used to synthesis electrochemical sensors for ascorbic acid [12], $\beta$-NADH $[12,13]$, $\mathrm{H}_{2} \mathrm{O}_{2}$ [13], hydrazine [14], glucose [15,16], dopamine [17,18], cadmium [19], Cytochrome c [20], chemicalwarfare agent [21], and others biosensors [22]. In order to disperse graphene in different solvents, for example, DMF, acetone, in this study, graphene were successfully dispersed into chitosan. Then the CS-dispersed graphene was fabricated. The CS-dispersed graphene film coated

*Corresponding author. onto the glassy carbon electrode, the electrochemical behaviors of analytes such as mitoxantrone were investigated using the CS-dispersed graphene film coated electrode, using a very sensitive and convenient voltampere method to determine mitoxantrone.

Mitoxantrone belongs to anthraquinone derivative anti-cancer drugs and its structure is given in Figure 1, which can be connected with DNA molecules, as a result of inhibiting the nucleic acid synthesis. Mitoxantrone has widely and efficient anti-cancer properties, it has been employed for the treatment of leukemia and breast cancer in clinical application. Therefore, the determination of mitoxantrone is very important. Until now, there are mainly three methods for detectting mitoxantrone as follows: chromatography [23,24], spectrophotometry [25],

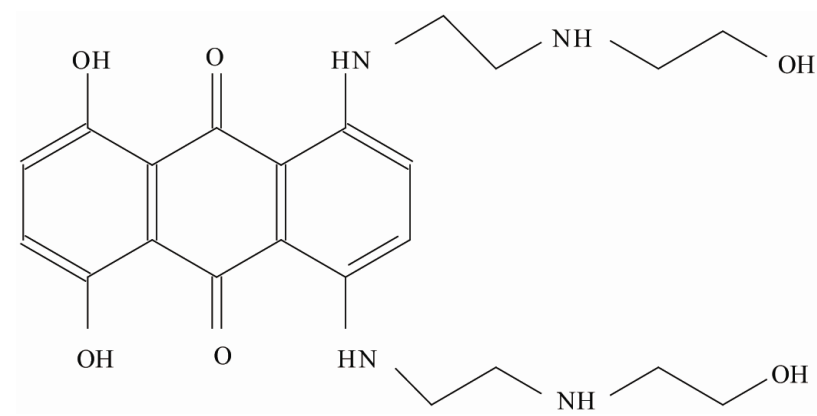

Figure 1. Chemical structure of mitoxantrone. 
electrochemical methods [26-29], however, the current methods are not sensitive enough, or equipments are expensive, which are difficult to promote. So we are longed for an effective, quick, inexpensive test method. This study suggests us the new procedure, which is suitted for the detection of mitoxantrone, has some advantages such as ultrasensitivity, rapid response and excellent reproducibility. A few years ago, the detection of mitoxantrone on ion implantation modified electrode had been reported that it had a high limit of detection $\left(1.8 \times 10^{-8}\right.$ $\mathrm{mol} / \mathrm{l})$. But the limit of detection of current methods is lower than that of the reported method.

\section{Experimental}

\subsection{Reagents and Apparatus}

The mitoxantrone was purchased from South Zhejiang Pharmaceutical Co., Ltd. A $1.0 \times 10^{-4} \mathrm{~mol} / \mathrm{l}$ standard solution of mitoxantrone was dissolved into $\mathrm{H}_{2} \mathrm{O}$ and kept in a refrigerator, $0.1 \mathrm{~mol} / \mathrm{l}$ sulfuric acid solution, some working standard solutions were prepared by dilution with water, and all the aqueous solutions were prepared with doubly distilled water. Graphite, hydrazine hydrate solution (85 wt\%) was purchased from Shanghai Chemical Reagent Co., Ltd. CS-dispersed graphene was fabricated from graphite. Chitosan (from Sinopharm Chemical Reagent Co., Ltd.) solution was prepared by dissolving chitosan in $1 \%$ acetic acid solution with magnetic stirring for $24 \mathrm{~h}$. All the reagents were of analytical grade.

A CHI 660A (Shanghai ChenHua Instrument Company) was used for the voltammetric determination, scanning electron microscope (SEM), and a traditional three-electrode system, CS-dispersed graphene modified electrode, a saturated calomel reference electrode and a platinum wire electrode were employed.

\subsection{Prepartion of Film Coated CS-Dispersed Graphene GCE}

The GC electrode was mechanically polished $0.05 \mu \mathrm{m}$ alumina slurry, then rinsed and sonicated in purified water, dried in the infrared lamp. The CS-dispersed graphene came to form a black suspension by ultrasonication agitation for approximately 2 hours. A certain volume of the CS-dispersed graphene solution was diluted to the appropriate concentration, was dropped onto a GC electrode and the electrode was allowed to dry in room temperature for $24 \mathrm{~h}$ to form a CS-graphene modified electrode, noted as CS-GR/GC electrode. Chitosan (CS)/ GC electrode was also constructed as above procedure except graphene in order to compare with the CS-GR/GC electrode.

\subsection{Procedure}

CS-dispersed graphene modified electrode scanned cyclic voltammograms for several times (scanning range from 0.50 to $0.90 \mathrm{~V}$ ) in a $4 \mathrm{ml}$ volume of $0.1 \mathrm{~mol} / \mathrm{l}$ sulfuric acid solution until the cyclic voltammograms curves were stable. And added an amount of mitoxantrone standard solution. The scanning range of differential pulse voltammograms from 0.60 to $0.85 \mathrm{~V}$ under open-circuit while stirring the for 2.5 mins that were recorded after 15 s quite time, the oxidation peak height was measured at about $0.74 \mathrm{~V}$. After each detection, the CS-dispersed graphene modified electrode scanned cyclic voltammetry in acid solution to remove substance which adsorbed on the electrode surface, as a result of activating electrode. The process of preparation modified electrode which is showed in Figure 2.

\section{Results and Discussion}

\subsection{SEM Characterization of Graphene}

The morphologies of graphene oxides, graphene were examined by SEM. The typical SEM imagines of graphene oxide (A) and the CS-dispersed graphene (B) on the electrode Surface which is showed in Figure 3, respectively. The morphology of graphene oxide is granular, but there is a small part of the sheet that can be seen from the Figure 2. However, the most of the CS-dispersed graphene film is seem to be a flake-like shape which is very different from the former. In addition, the CS-dispersed graphene was fabricated through a simple drop casting method on GCE exhibits a layer structure.

\subsection{Electrochemical Responses of Mitoxantrone}

The voltammetric behavior of mitoxantrone at the bare GCE is investigated using cyclic voltammetry from 0.50 to $0.90 \mathrm{~V}$ and the results show that there is no obvious oxidation or reduction responses of mitoxantrone. The voltammetric behavior of mitoxantrone at the CS-dispersed graphene film coated electrode is also investigated by CV. The CV from 0.50 to $0.90 \mathrm{~V}$ of a CS-dispersed graphene film-modified electrode in a $\mathrm{pH} 1.0$ Sulfuric acid solution (without mitoxantrone) is shown as curve a in Figure 4, and no observable peak appears. When the concentration of mitoxantrone is $1.0 \times 10^{-6} \mathrm{~mol} / \mathrm{l}$, not only appears an oxidation peak at $0.81 \mathrm{~V}$ from 0.50 to $0.90 \mathrm{~V}$, but also appears a corresponding reduction peak on the reverse scan at $0.72 \mathrm{~V}$ from 0.50 to $0.90 \mathrm{~V}$, suggesting that the electrode reaction of mitoxantrone at the CS-dispersed graphene film is possible reversible.

The voltammetric responses of mitoxantrone have been compared by differential pulse voltammogram (DPV) which the results are showed in Figure 5. Both bare GCE (Figure 5(a)) and chitosan-film coated GCE (Figure 

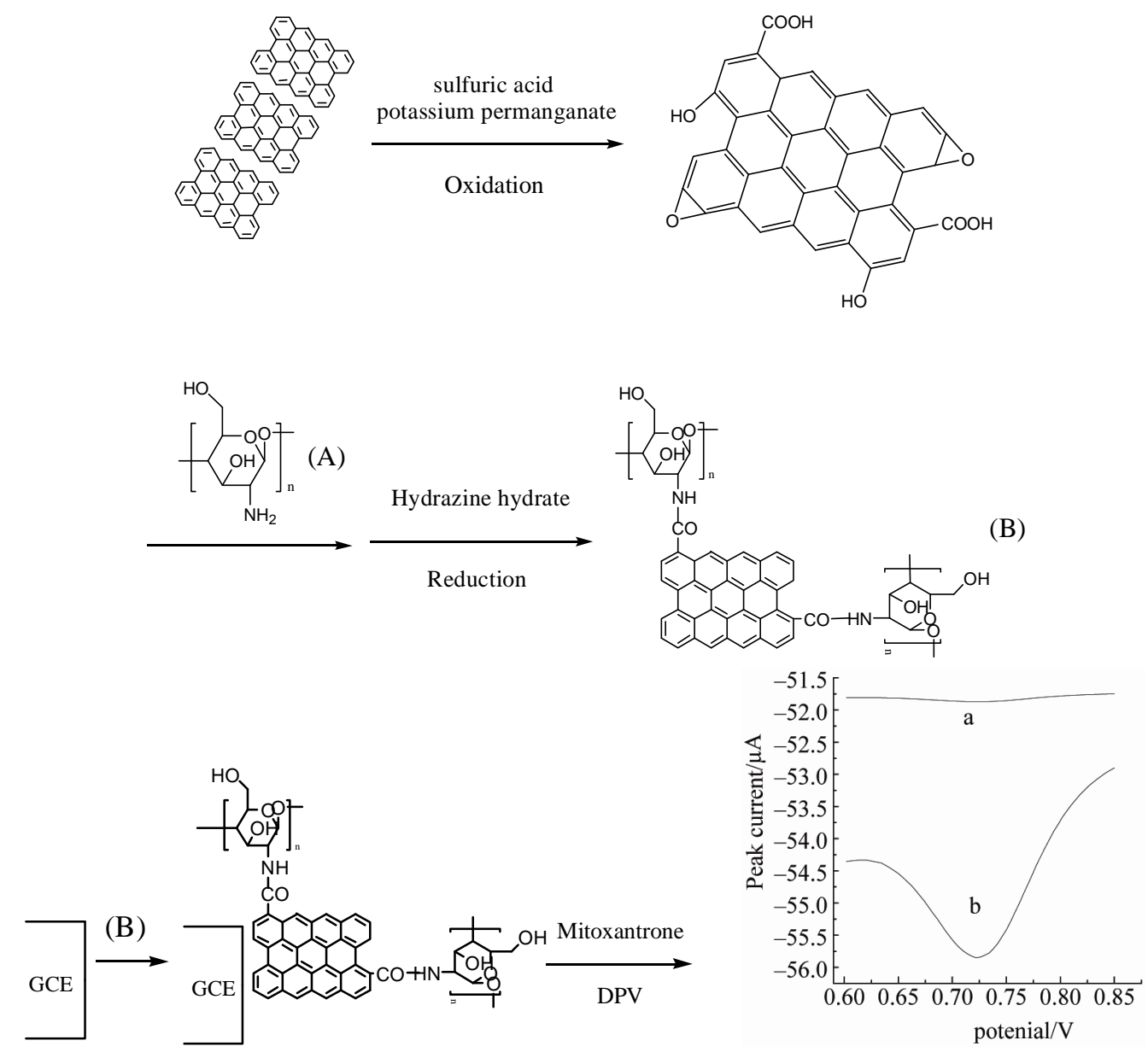

Figure 2. The process of preparation modified electrode A is chitosan, B is CS-dispersed graphene, curve a is a bare GCE, but curve $b$ is a CS-dispersed graphene film coated GCE.

5(b)) are no observable oxidation current when the concentration of mitoxantrone is $1.0 \times 10^{-8} \mathrm{~mol} / \mathrm{l}$. But under the identical conditions, mitoxantrone appears a very significant oxidation signal whose potential is $0.74 \mathrm{~V}$ at the CS-dispersed graphene film coated GCE (Figure 5(c)). The significant oxidation peak current is undoubtedly attributed to characteristics of graphene. These suggested that graphene has some special electrochemical properties and big specific surface area which could provide much more reaction sites for the electrochemical oxidation of mitoxantrone, then promote the exchange rates of mitoxantrone, which results in increasing the oxidation peak current greatly.

\subsection{Effect of Supporting Electrolyte}

To optimize the determination conditions of mitoxantrone, many support electrolytes were also tested, such as $\mathrm{HCl}, \mathrm{H}_{2} \mathrm{SO}_{4}, \mathrm{HAc}-\mathrm{NaAc}, \mathrm{KCl}$, phosphate buffer, B-R buffer, acetic acid-acetate buffer, others were not as suitable as Sulfuric acid solution for the determination.

The $\mathrm{pH}$ value of the base solution has an important influence on the oxidation of mitoxantrone at the GCE modified CS-dispersed graphene. The $\mathrm{pH}$ effect was studied from 0 to 7.0. It showed that the highest oxidation peak current of mitoxantrone was obtained at $\mathrm{pH} 1.0$. Hence, the Sulfuric acid solution $\mathrm{pH}$ of 1.0 was supporting electrolyte for determining mitoxantrone.

\subsection{Accumlation Conditions}

Accumlation conditions can affect the detection sensitivity. Accumlation time and accumlation potential are two important factors for the accumlation step. The effect on peak current response from accumulation potential and time which is studied by DPV, the concentration of mitoxantrone used was $1.0 \times 10^{-8} \mathrm{~mol} / \mathrm{l}$.

When accumulation potential is shifted from +0.5 to $-0.5 \mathrm{~V}$, the peak current keep stable. So accumulation at open circuit is adopted. The peak current increases sharply with increasing accumulation time which is illustrated in Figure 6. The value of peak current reaches its maximum at 2.5 mins, and then levels off. This may be the electrode surface become saturated. Thus, 2.5 mins under opencircuit is generally chosen as accumulation condition. 

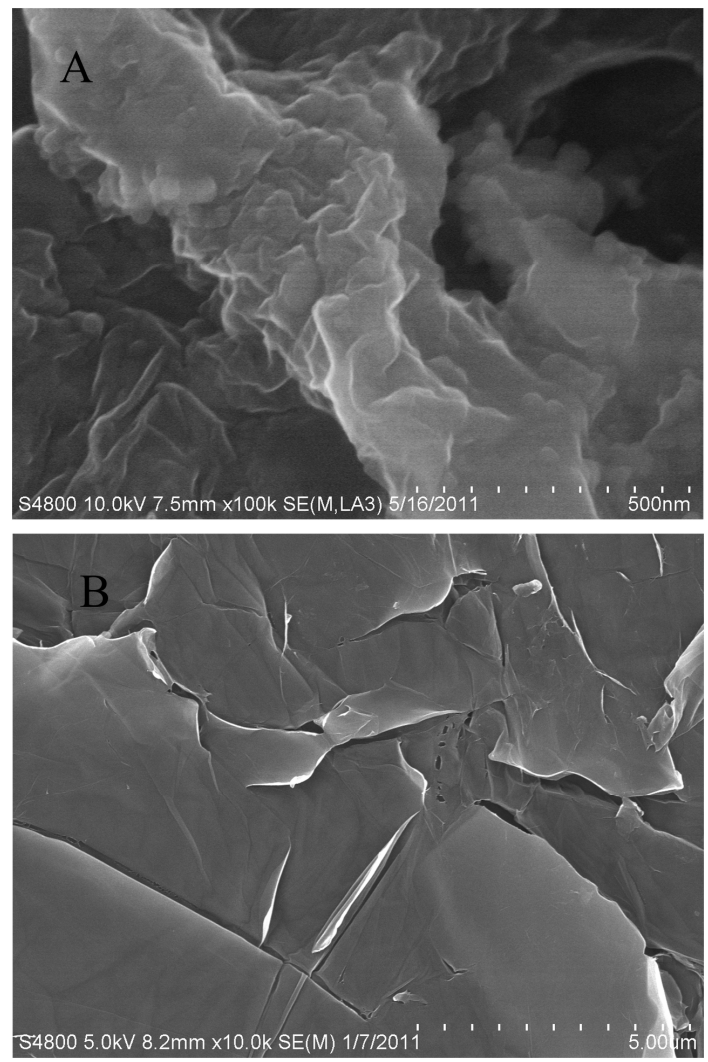

Figure 3. SEM images of: (A) GO and (B) CS-dispersed graphene.

\subsection{The Amount of CS-Dispersed Graphene Dispersion on the GCE}

The amount of CS-dispersed graphene dispersion on the

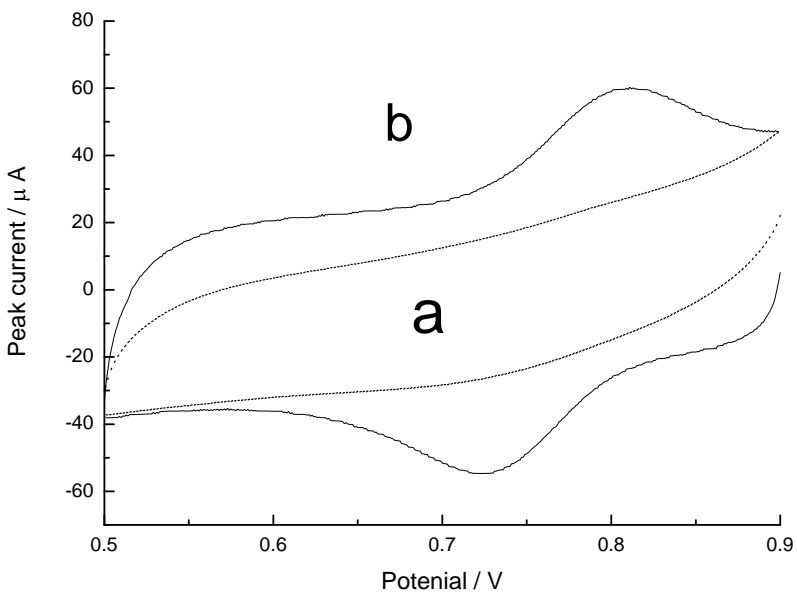

Figure 4. Cyclic voltammograms of a CS-dispersed graphene film coated GCE. Curve (a) in pH 1.0 Sulfuric acid solution; curve (b) a $+1.0 \times 10^{-6} \mathrm{~mol} / \mathrm{l}$ of mitoxantrone. Scan rate: $100 \mathrm{mV} / \mathrm{s}$.

GCE surface determines the thickness of the cast film. The amount of CS-dispersed graphene dispersion on the GCE surface determines the thickness of the cast film. The amount of CS-dispersed graphene has influence on the peak current which is illustrated in Figure 7. When the amount is from 0 to $8 \mu \mathrm{l}$ which the peak current increases remarkably with the increasing volumes of CSdispersed graphene suspension. But the peak current is more stable and higher when the amount is from 8 to 30 $\mu \mathrm{l}$, after that amount, it decreases. It has some relationship with the thickness of the film. If the film was too thin, the mitoxantrone amount adsorbed was small which resulting in the small peak current. But when the film

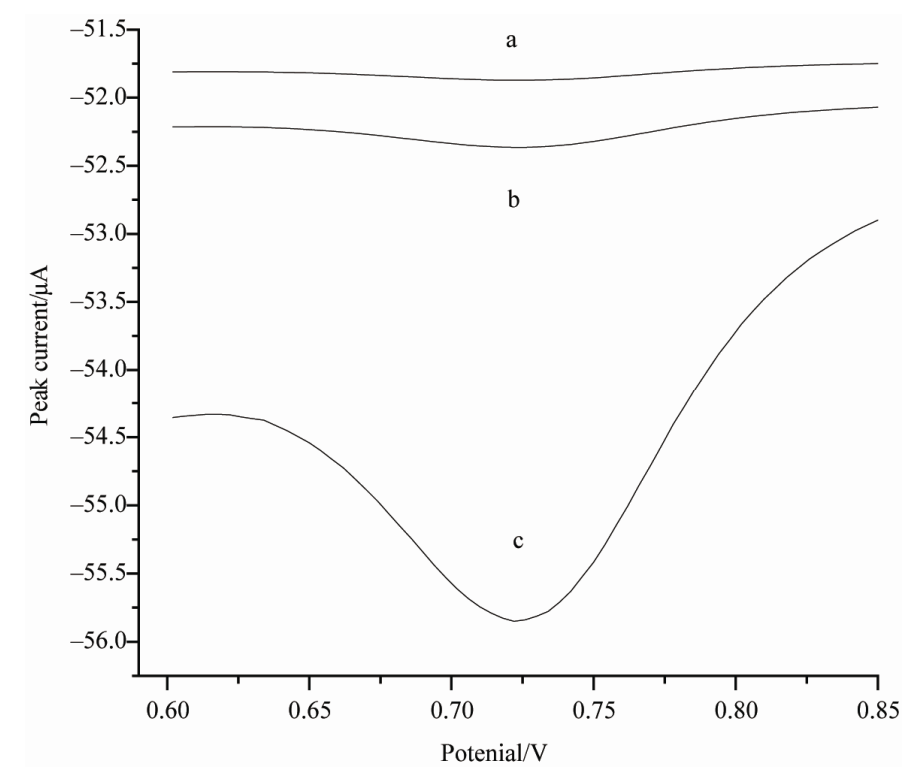

Figure 5. Differential pulse voltammograms of $1.0 \times 10^{-8} \mathrm{~mol} / \mathrm{l}$ of mitoxantrone at different electrodes. Curve (a) a bare GCE; curve (b) a chitosan (CS)-film coated GCE; curve (c) a CS-dispersed graphene film coated GCE. Accumulation times = 2.5 mins; pulse amplitudes $=50 \mathrm{mV}$, scan rates $=100 \mathrm{mV} / \mathrm{s}$, pulse widths $=50 \mathrm{~ms}$. 


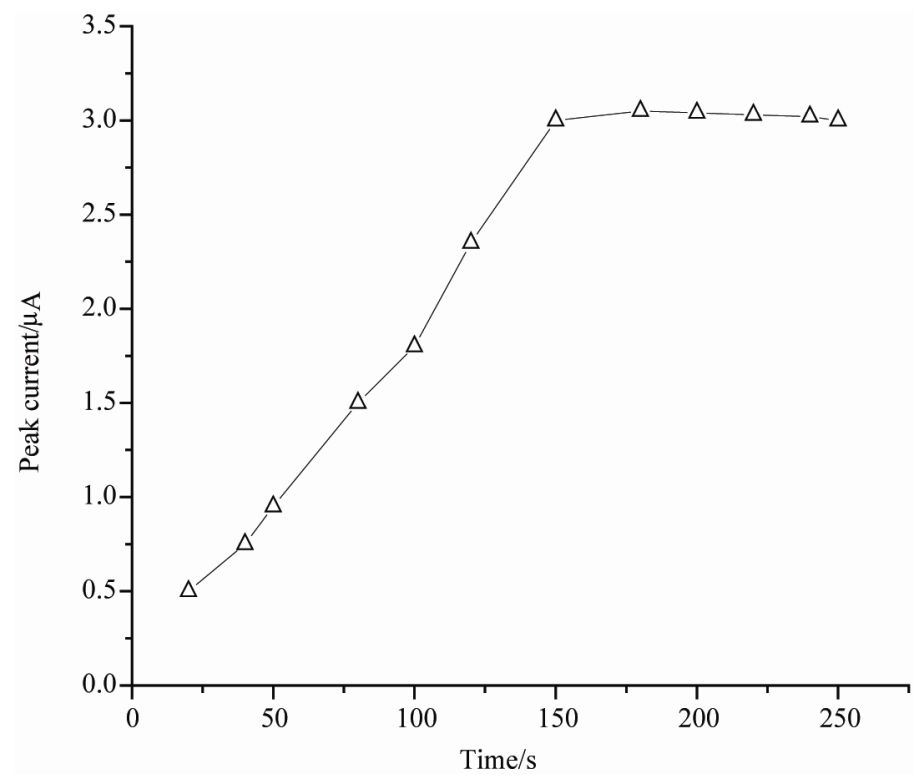

Figure 6. Influences of accumulation time on the oxidation peak current of $1 \times 10^{-8} \mathrm{~mol} / \mathrm{l}$ of mitoxantrone at the CS-dispersed graphene film coated the GCE conditions are the same as in Figure 5.

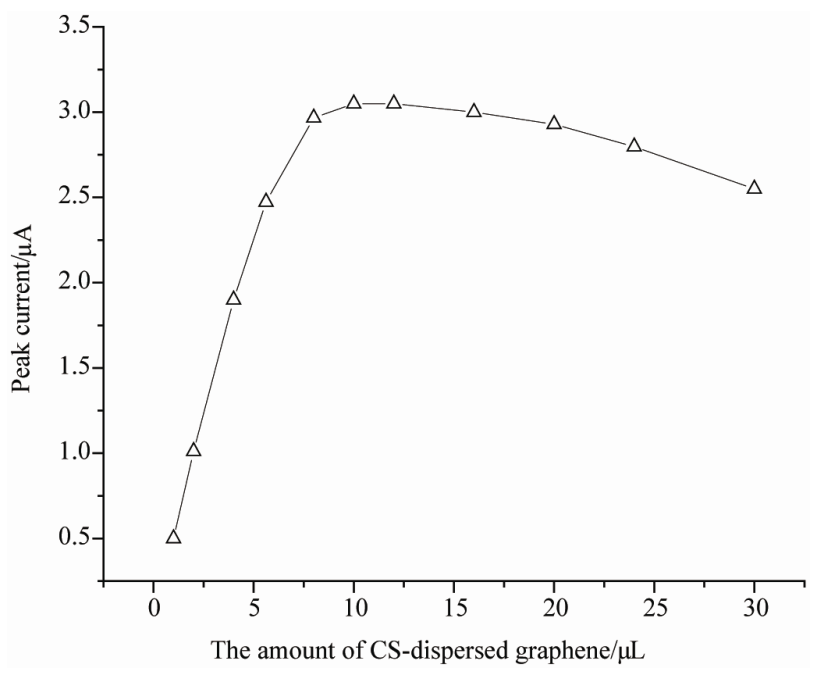

Figure 7. Effects of amounts of CS-dispersed graphene suspension on the oxidation peak current of $1 \times 10^{-8} \mathrm{~mol} / \mathrm{l}$ of mitoxantrone. Other conditions are the same as in Figure 5.

was too thick, the film conductivity reduced and the film became not so stable as CS-dispersed graphene, so the peak current decreases. Therefore, $8 \mu \mathrm{l}$ CS-dispersed graphene suspension solution was used in this study.

\subsection{Influence of Scan Rate}

The influence of scan rate on mitoxantrone oxidation at the CS-dispersed graphene was investigated by cyclic voltammetry which is showed in Figure 8. At scan rates in the range from 10 to $500 \mathrm{mV} / \mathrm{s}$. The oxidative peak current of the CS-dispersed graphene in mitoxantrone solution increases linearly with the scan rate, therefore, the contribution of adsorption played a more important role in the electrode process. From the symmetry of oxidation peak and reduction peaks which indicates oxidation and reduction of mitoxantrone on surface of the CSdispersed grapheme modified electrodes is not a completely reversible process. The peak potential has a slight change with the increasing scan rates, thus, mitoxantrone on the CS-dispersed grapheme is the quasi-reversible nature of the electrode reaction. The mechanism for the oxidative peak process may be written as in Figure $\mathbf{9}$.

In summary, when the scan rates go up, peak current increases, reversible becomes worse, peak shape becomes wide, at the same time considering signal-noise ratio and other factors, so the best scan rates is $100 \mathrm{mV} / \mathrm{s}$.

\subsection{Calibration Graph and Stability}

The relationships between the oxidation peak current and the concentration of mitoxantrone were examined by using DPV which were demonstrated in Figure 10. When the range is $1 \times 10^{-6}-1 \times 10^{-7} \mathrm{~mol} / \mathrm{l}, 2 \times 10^{-8}-8 \times 10^{-8}$ $\mathrm{mol} / \mathrm{l}, 8 \times 10^{-9}-6 \times 10^{-10} \mathrm{~mol} / \mathrm{l}$, oxidation peak current (y) and the concentration of mitoxantrone (x) have a good linear relationship. Their linear equations and correlation coefficients as follow: $\mathrm{y}=1.099+19.03 \mathrm{x}, \mathrm{r}=0.9957$; $\mathrm{y}=$ $5.482+0.1764 x, r=0.9903 ; y=12.15+0.06702 x, r=$ 0.9924 . And its detection limit is $2 \times 10^{-10} \mathrm{~mol} / \mathrm{l}$.

The relative standard deviation of $3.7 \%$ for ten parallel determinations in mitoxantrone were studied by the same modified electrode which exhibited good repeatability and reproducibility, after two weeks, the average peak current of the modified electrodes only increased $4.5 \%$ from 20 parallel determinations. However, the peak po- 


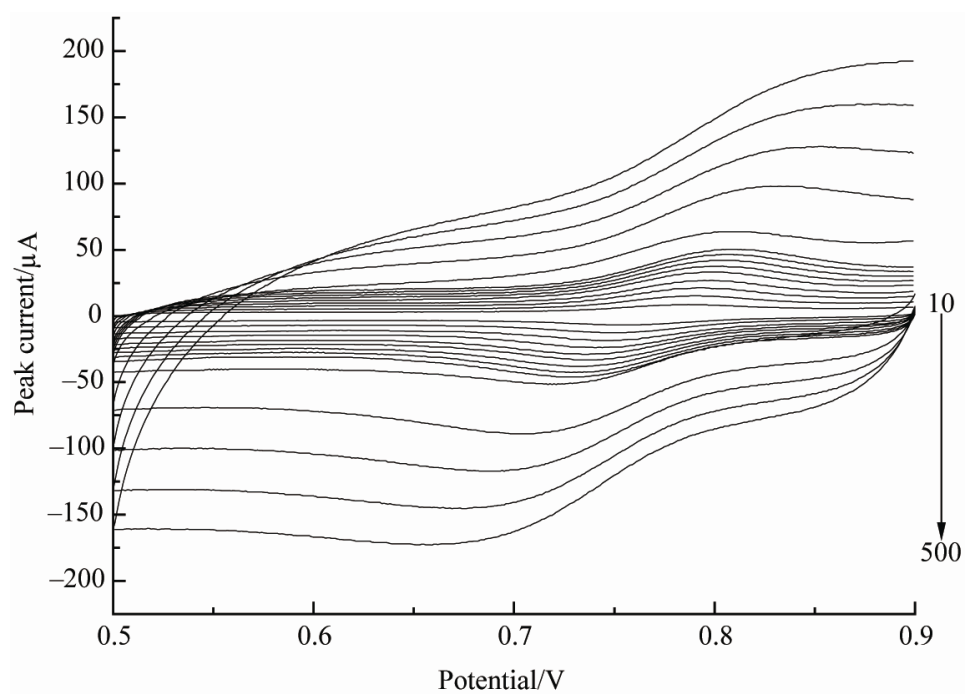

Figure 8. Cyclic voltammograms of mitoxantrone at CS-dispersed graphene modified electrode with scan rates (from inner to outer): 10, 20, 30, 40, 50, 60, 70, 80, 90, 100, 200, 300, 400, $500 \mathrm{mV} / \mathrm{s}$. respectively.
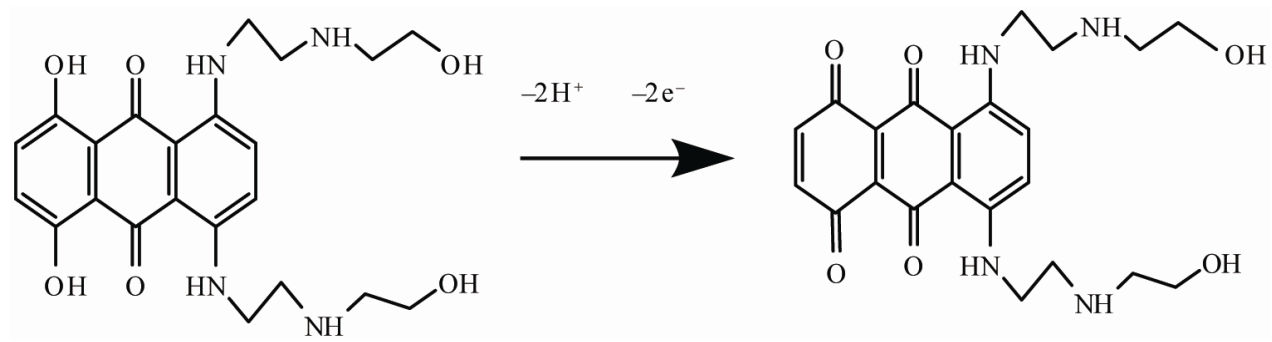

Figure 9. The mechanism for the oxidative peak process.

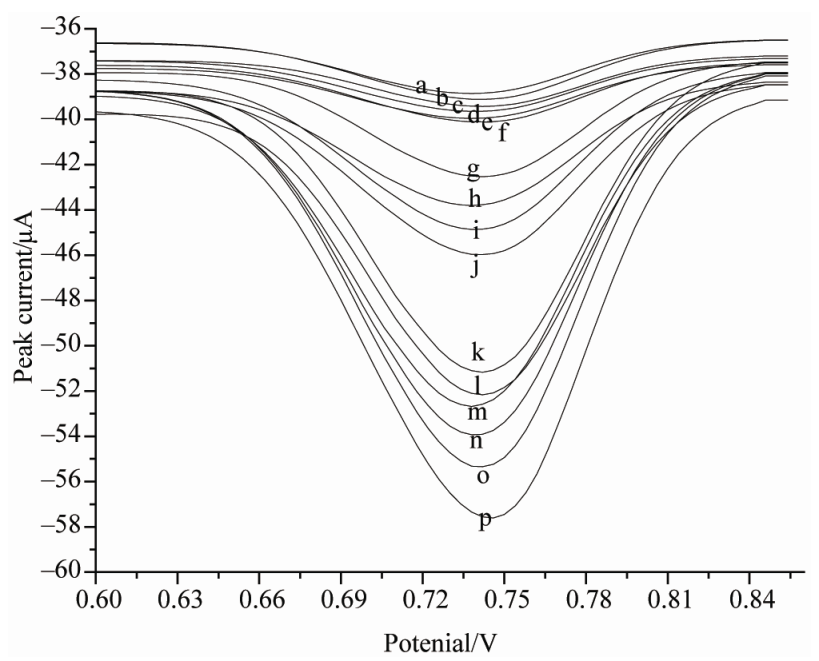

Figure 10. Differential pulse voltammograms (DPV) of different concentration values of Actinomycin D (ACTD) at CS-dispersed grapheme modified GCE (from a to p): 0.08, 0.06, 0.2, 0.4, 0.6, 0.8, 2.0, 4.0, 6.0, 8.0, 10.0, 20.0, 40.0, 60.0, 80.0, $100.0\left(\times 10^{-8} \mathrm{~mol} / \mathrm{L}\right)$. Conditions are the same as in Figure 5.

tential was almost constant which the modified electrodes shows good stability.

\subsection{Interferences}

Interferences of some organic compounds influenced the peak oxidation current of mitoxantrone solution which was detected by cyclic voltammetry. Some common organic compounds in human body, such as urea, glucose, VC, etc. The concentration of mitoxantrone solution is 1 $\times 10^{-6} \mathrm{~mol} / \mathrm{l}$, while the concentration of interfering substances is 100 times of the concentration of base solution. The result indicate that CS-dispersed grapheme affect the detection of mitoxantrone hardly that in the presence of urea, glucose, VC. Thus, CS-dispersed graphene modified electrode has good selectivity in detectting mitoxantrone.

\subsection{Determination of Mitoxantrone in Urine Samples}

According to the observation above, it is found that the CS-dispersed grapheme modified GCE has a high sensitivity and selectivity in the laboratory. The CS-dispersed grapheme modified GCE was used to detect urine samples which contained mitoxantrone (supported by Jiaxingfirst hospital) in order to test its practical application. Mitoxantrone was diluted to a concentration in appropriate 
Table 1. Determination of mitoxantrone in urine samples.

\begin{tabular}{cccc}
\hline Sample & Ideal concentration (mol/l) & Found (mol/l) & Recovery (\%) \\
\hline 1 & $6.24 \times 10^{-8}$ & $6.08 \times 10^{-8}$ & 97.4 \\
2 & $6.59 \times 10^{-8}$ & $6.76 \times 10^{-8}$ & 102.6 \\
3 & $6.80 \times 10^{-8}$ & $6.81 \times 10^{-8}$ & 100.1 \\
4 & $12.89 \times 10^{-8}$ & $12.67 \times 10^{-8}$ & 98.3 \\
\hline
\end{tabular}

linear range by doubly distilled water, then determine the content of mitoxantrone in urine samples under the best conditions, the results are demonstrated in Table 1, each sample were parallel determined for three times, the recovery are in range from $97.4 \%$ to $102.6 \%$.

\section{Conclusion}

Graphene were dispersed into chitosan which began to obtain a stable and homogeneous CS-dispersed graphene suspension. And finally the CS-dispersed graphene filmcoated electrode was fabricated. Because there are some unique advantages exist in graphene, for instance, incredible electronic properties, big specific surface area, strong adsorptive capacity, high migration rate and good thermal conductivity rate, excellent mechanical and optical-electrical properties. Graphene is used in modified electrode and has a good prospect in analysis determination. And then a very sensitive and simple electrochemical method was developed to detect mitoxantrone in real samples.

\section{Acknowledgements}

This work has been supported by the National Natural Science Foundation of China (20275034), the Natural Science Foundation of Zhejiang Province (Y405468), Science and Technology Projects of Zhejiang Province (2007F70008) and Science and Technology Projects of Jiaxing (2008AY2017).

\section{REFERENCES}

[1] K. S. Novoselov, A. K. Gein, S. V. Morozov, D. Jiang, Y. Zhang, S. V. Dubonos, I. V. Grigorieva and A. A. Firsov, "Electric Field Effect in Atomically Thin Carbon Films," Science, Vol. 306, No. 5696, 2004, pp. 666-669. doi:10.1126/science.1102896

[2] A. K. Geim and K. S. Novoselov, "The Rise of Graphene," Nature Materials, Vol. 6, 2007, pp. 183-191. doi:10.1038/nmat1849

[3] C. N. R. Rao, A. K. Sood, K. S. Subrahmanyam and A. Govindaraj, "Graphene: The New Two-Dimensional Nanomaterial," Angewandte Chemie International Edition, Vol. 48, No. 42, 2009, pp. 7752-7777. doi:10.1002/anie.200952249

[4] M. Pumera, "Electrochemistry of Graphene: New Horizons for Sensing and Energy Storage,” The Chemical Re- cord, Vol. 9, No. 4, 2009, pp. 211-223. doi:10.1002/tcr.200900008

[5] W. Yang, K. R. Ratinac, S. P. Ringer, P. Thordarson, J. J. Gooding and F. Braet, "Carbon Nanomaterials in Biosensors: Should You Use Nanotubes or Graphene?” Angewandte Chemie International Edition, Vol. 49, No. 12, 2010, pp. 2114-2138. doi:10.1002/anie.200903463

[6] D. Li, M. B. Muller, S. Gilje, R. B. Kaner and G. G. Wallace, "Processable Aqueous Dispersions of Grapheme Nanosheets,” Nature Nanotechnology, Vol. 3, 2008, pp. 101-105. doi:10.1038/nnano.2007.451

[7] S. Stankovich, D. A. Dikin, G. H. B. Dommett, K. M. Kohlhaas, E. J. Zimney, E. A. Stach, R. D. Piner, S. T. Nguyen and R. S. Ruoff, "Graphene-Based Composite Materials,” Nature, Vol. 442, 2006, pp. 282-286. doi:10.1038/nature04969

[8] Y. X. Xu, H. Bai, G. W. Lu, C. Li and G. Q. Shi, "Flexible Graphene Films via the Filtration of Water-Soluble Noncovalent Functionalized Graphene Sheets,” Journal of the American Chemical Society, Vol. 130, No. 18, 2008, pp. 5856-5857. doi:10.1021/ja800745y

[9] R. Muszynski, B. Seger and P. V. J. Kamat, "Decorating Graphene Sheets with Gold Nanoparticles,” The Journal of Physical Chemistry C, Vol. 112, No. 14, 2008, pp. 5263-5266. doi:10.1021/jp800977b

[10] C. M. Chen, Q.-H. Yang, Y. G. Yang, W. Lv, Y. F. Wen, P.-X. Hou, M. Z. Wang and H.-M. Cheng, "Self-Assembled Free-Standing Graphite Oxide Membrane,” Advanced Materials, Vol. 21, No. 29, 2009, pp. 3007-3011. doi:10.1002/adma.200803726

[11] H. Q. Chen, M. B. Muller, K. J. Gilmore, G. G. Wallace and D. Li, "Mechanically Strong, Electrically Conductive, and Biocompatible Graphene Paper," Advanced Materials, Vol. 20, No. 18, 2008, pp. 3557-3561.

doi:10.1002/adma.200800757

[12] J. W. Wang, S. L. Yang, D. Y. Guo, P. Yu, D. Li, J. S. Ye and L. Q. Mao, "Comparative Studies on Electrochemical Activity of Graphene Nanosheets and Carbon Nanotubes," Electrochemistry Communications, Vol. 11, No. 10, 2009 pp. 1892-1895. doi:10.1016/j.elecom.2009.08.019

[13] W. J. Lin, C. S. Liao, J. H. Jhang and Y. C. Tsai, “Graphene Modified Basal and Edge Plane Pyrolytic Graphite Electrodes for Electrocatalytic Oxidation of Hydrogen Peroxide and Beta-Nicotinamide Adenine Dinucleotide," Electrochemistry Communications, Vol. 11, No. 11, 2009, pp. 2153-2156. doi:10.1016/j.elecom.2009.09.018

[14] Y. Wang, Y. Wan and D. Zhang, "Reduced Grapheme Sheets Modified Glassy Carbon Electrode for Electrocatalytic Oxidation of Hydrazine in Alkaline Media,” Electrochemistry Communications, Vol. 12, No. 2, 2010, pp. 187190. doi:10.1016/j.elecom.2009.11.019

[15] X. P. Chen, H. Z. Ye and W. Z. Wang, "Electrochemiluminescence Biosensor for Glucose Based on Graphene/ Nafion/GOD Film Modified Glassy Carbon Electrode," Electroanalysis, Vol. 20, No. 20, 2010, pp. 2347-2352. doi:10.1002/elan.201000095

[16] P. Wu, S. A. Qian and Y. J. Hua, "Direct Electrochemistry of Glucose Oxidase Assembled on Graphene and Application to Glucose Detection,” Electrochimica Acta, Vol. 
55, No. 28, 2010, pp. 8606-8614. doi:10.1016/j.electacta.2010.07.079

[17] Y. Wang, Y. M. Li and L. H. Tang, “Application of Graphene-Modified Electrode for Selective Detection of Dopamine," Electrochemistry Communications, Vol. 11, No. 4, 2009, pp. 889-892. doi:10.1016/j.elecom.2009.02.013

[18] L. Tan, K.-G. Zhou, Y. H. Zhang, et al., "Nanomolar Detection of Dopamine in the Presence of Ascorbic Acid at Beta-Cyclodextrin/Graphene Nanocomposite Platform," Electrochemistry Communications, Vol. 12, No. 4, 2010, pp. 557-560. doi:10.1016/j.elecom.2010.01.042

[19] J. Li, S. J. Guo and Y. M. Zhai, "Nafion-Graphene Nanocomposite Film as Enhanced Sensing Platform for Ultrasensitive Determination of Cadmium," Electrochemistry Communications, Vol. 11, No. 5, 2009, pp. 1085-1088. doi:10.1016/j.elecom.2009.03.025

[20] J.-F. Wu, M.-Q. Xu and G.-C. Zhao, "Graphene-Based Modified Electrode for the Direct Electron Transfer of Cytochrome c and Biosensing," Electrochemistry Communications, Vol. 12, No. 1, 2010, pp. 175-177. doi:10.1016/j.elecom.2009.11.020

[21] J. T. Robinson, F. K. Perkins, E. S. Snow, Z. Wei and P. E. Sheehan, "Reduced Graphene Oxide Molecular Sensors,” Nano Letters, Vol. 8, No. 10, 2008, pp. 3137-3140. doi:10.1021/nl8013007

[22] M. Zhou, Y. M. Zhai and S. J. Dong, "Electrochemical Sensing and Biosensing Platform Based on Chemically Reduced Graphene Oxide,” Analytical Chemistry, Vol. 81, No. 14, 2009, pp. 5603-5613. doi:10.1021/ac900136z

[23] K. M. Rentsch, R. A. Schwendener and E. Hänseler,
"Determination of Mitoxant rone in Mouse Whole Blood and Different Tissues by High-Performance Liquid Chromatography," Journal of Chromatography B: Biomedical Sciences and Applications, Vol. 679, No. 1-2, 1996, pp. 185-192. doi:10.1016/0378-4347(96)00023-0

[24] P. Guo, L. M. Ye, W. Z. Wu and T. S. Wu, "Determination of Antitumour Drug Mitoxantrone in Plasma Using HPLC Column Switching Technique,” Acta Pharmaceutica Sinica, Vol. 26, No. 5, 1991, pp. 367-369.

[25] Q. Z. Zhou, C. Y. Wu, L. K. Zhang, N. Li and X. Y. He, "Determination of Mitoxantrone by Spectrophotometry," Chinese Journal of Pharmaceutical Analysis, Vol. 17, No. 6, 1997, pp. 403-405.

[26] H. Z. Song, M. F. Yang and Z. Y. Gu, "Adsorptive Behaviour of Mitoxantrone and Its Adsorptive Voltammeteic Determination," Chinese Journal of Analytical Chemistry, Vol. 21, 1993, pp. 1285-1287.

[27] M. D. Guo, "Study on Mitoxantrone Using Mercury Film Carbon Fiber Microelectrode by 1.5 Order Differential Stripping Voltammetry," Chinese Analytical Sciences Acta, Vol. 11, 1995, pp. 46-48.

[28] J. B. Hu and Q. L. Li, "Studies on the Voltammetric Behavior of Mitoxantrone and Its Application at the Ni/GC Modified Electrode," Chemical Journal of Chinese Universities, Vol. 22, No. 3, 2001, pp. 380-384.

[29] M. O. Brett, T. R. A.Macedo, D. Raimundo, M. H. Marques and S. H. P. Serrano, "Electrochemical Oxidation of Mitoxantrone at a Glassy Carbon Electrode,” Analytica Chimica Acta, Vol. 385, No. 1-3, 1999, pp. 401-408. doi:10.1016/S0003-2670(98)00807-1 